\title{
Laparoscopic Repair of a Ruptured Diaphragm: Avoiding a Trauma Laparotomy
}

\author{
Kenneth L. Wilson, MD, Erin B. Bowman, MD, Leslie R. Matthews, MD, Omar K. Danner, MD, \\ James C Rosser, Jr, MD \\ Department of Surgery, Hurley Medical Center, Michigan State University, Flint, Michigan, USA (Dr Wilson). \\ Department of Surgery, Morehouse School of Medicine, Atlanta, Georgia, USA (Drs Bowman, Matthews, Danner). \\ Department of Surgery, Florida Celebration Hospital, Celebration Florida, USA (Dr Rosser).
}

\begin{abstract}
Background: A traumatic diaphragm rupture presents a unique obstacle to a minimally invasive surgical approach; most repairs are performed during an emergency laparotomy. Diaphragm injuries are diagnosed in the acute phase of blunt-force trauma in only $10 \%$ of cases, and a high index of suspicion must be maintained to avoid strangulation of the abdominal organs that have herniated into the thoracic cavity. A laparoscopic evaluation and repair of an acute blunt-force rupture of the diaphragm can be diagnostic and curative, mimicking the outcome of an open procedure.

Case Description: A 23-year-old woman had a left-side blunt-force rupture of the diaphragm sustained in a high-impact motor vehicle collision. The focused assessment with sonography for trauma (FAST) was negative. The survey chest radiograph identified only streaky opacities that were read as atelectasis. Computed tomography of the abdomen revealed the presence of a congenital abnormality versus a ruptured diaphragm. A diagnostic trauma laparoscopy was performed to evaluate for the possibility of a left-side rupture, and at that point, the spleen and the stomach were found to be located in the left chest, herniating through a rupture in the left diaphragm. A grade I splenic laceration was present. The abdominal structures were reduced and the traumatic rupture was successfully repaired laparoscopically.

Discussion: Traumatic rupture of the left diaphragm can occur as an occult injury after blunt-force trauma to the torso. The liver lends protection to the diaphragm and a right-side rupture is far less common than one on the left side. The initial diagnostic plain chest x-ray may not reveal the tear in the diaphragm and the herniation of abdominal viscera into the thoracic cavity. Laparoscopy has been used to evaluate the possibility of a rent in the diaphragm when the patient is hemodynamically stable and the diagnosis is uncertain. Although initial laparoscopic or thorascopic evaluation of a potential rupture of the diaphragm is the standard of care in the trauma literature, laparoscopic repair is not widely accepted. However, laparoscopic evaluation of acute torso trauma with reduction of abdominal viscera and subsequent laparoscopic repair of the diaphragm can be successful.
\end{abstract}

Key Words: Blunt abdominal trauma, Laparoscopic diaphragmatic hernia repair, Ruptured diaphragm.

Citation Wilson KL, Bowman EB, Matthews LR, Danner OK, Rosser JC. Laparoscopic repair of a diaphragmatic injury: avoiding a trauma laparotomy. CRSLS e2015.00011. DOI: 10.4293/CRSLS.2015.00011.

Copyright (c) 2015 by SLS, Society of Laparoendoscopic Surgeons. This is an open-access article distributed under the terms of the Creative Commons Attribution-Noncommercial-ShareAlike 3.0 Unported license, which permits unrestricted noncommercial use, distribution, and reproduction in any medium, provided the original author and source are credited.

Address correspondence to: Kenneth L. Wilson, MD, Department of Surgery, Hurley Medical Center, Michigan State University, One Hurley Plaza, Flint, MI, 48503. Telephone: 810-262-6689, Fax: 810-262-6341, E-mail: kwilson1@hurleymc.com.

\section{INTRODUCTION}

Blunt-force injury of the diaphragm is a rare condition, and the diagnosis is easily missed, requiring a high index of suspicion. The overall incidence is $0.8 \%$ to $5.8 \%$ in blunt-force trauma, $2.5 \%$ to $5 \%$ in blunt abdominal trauma, and $1.5 \%$ in blunt thoracic trauma. ${ }^{1,2}$ Laparoscopy may be used successfully for both diagnosis and repair, but there are very few reports about laparoscopic diaphragmatic hernia repair. ${ }^{3}$ Laparoscopy has been used to evaluate 
occult penetrating injuries to the thoracoabdominal region, because injuries associated with a lack of clinical and radiographic evidence on the left side are missed as much as $24 \%$ of the time; $18 \%$ of patients with a normal chest radiograph have diaphragm injuries. ${ }^{4,5}$ The first laparoscopic repair of a diaphragm injury was performed in 1994 for a penetrating injury, but the widely accepted treatment is by trauma laparotomy. ${ }^{6}$ Blunt-force trauma to the diaphragm is considerably more difficult to diagnose when the presentation is that of a hemodynamically stable patient, and the focused assessment with sonography for trauma (FAST) and initial chest radiograph are both interpreted as normal. We present a laparoscopic evaluation of an occult injury of the diaphragm, and the subsequent reduction of abdominal viscera with laparoscopic repair of a blunt-force injury to the left diaphragm.

\section{CASE REPORT}

A 23-year old woman was admitted to our level I trauma center after a motor vehicle accident, in which she was a restrained front seat passenger when the car was struck by another vehicle along the right front door panel. The FAST was negative. The chest radiograph findings were significant for streaky opacities present in the left base, representing atelectasis. There were no left-side rib fractures, and the appearance of both diaphragms was normal. Computed tomography (CT) of the abdomen suggested a congenital abnormality of the left diaphragm with the possibility of a diaphragm injury without elevation of either diaphragm. A diagnostic laparoscopy with subsequent laparoscopic repair of the left hemidiaphragm was performed.

\section{Laparoscopic Repair: Technical Considerations}

Pneumoperitoneum was established with the Hasson technique. A $30^{\circ}$ endoscope placed through the umbilical port was used to guide the remaining ports needed to perform the operation. A 5-mm trocar was placed at the right lateral edge of the rectus in the upper quadrant. A $12-\mathrm{mm}$ port for the surgeon's right hand was placed at the lateral edge of the right rectus, adjacent to the umbilicus, to allow the passage of a needle driver. A 5-mm left lateral subcostal port was placed to allow for retraction and reduction of abdominal viscera by the assistant.

\section{Procedure}

Initial abdominal exposure and exploration. A systematic exploration of the abdomen was performed quad- rant by quadrant, to identify the structures most likely to be injured during blunt abdominal trauma. The primary surgeon stood to the left of the patient, using the right-side ports for the exploration of the left diaphragm. The evaluation of the left upper quadrant revealed a disruption of the left hemidiaphragm, with the spleen herniated completely into the left thorax and the stomach and omentum resting in the lower portion of the left thoracic cavity. The completed survey of the abdomen elucidated a grade I splenic laceration and a left-side rupture of the diaphragm.

Repair of the diaphragm. In an effort to see the full extent of the tear in the diaphragm and the injury to the spleen, we placed a 5-mm grasper through the left upper quadrant port and placed gentle traction on the body of the stomach, allowing simultaneous reduction of the stomach, omentum, and spleen (Figure 1). The basis for acutely repairing the diaphragm is to reduce the abdominal contents and prevent strangulation and necrosis of the intra-abdominal contents chronically herniated into the thoracic cavity. Careful inspection was facilitated by the assistant's downward caudal compression of the spleen, stomach, and omentum. The lateral edge of the diaphragm had a "swiss cheese" appearance. The freshly debrided edges were held in apposition, demonstrating that a tension-free repair could be accomplished (Figure 2). The preferred method of repair for the operating surgeon is to use nonabsorbable interrupted

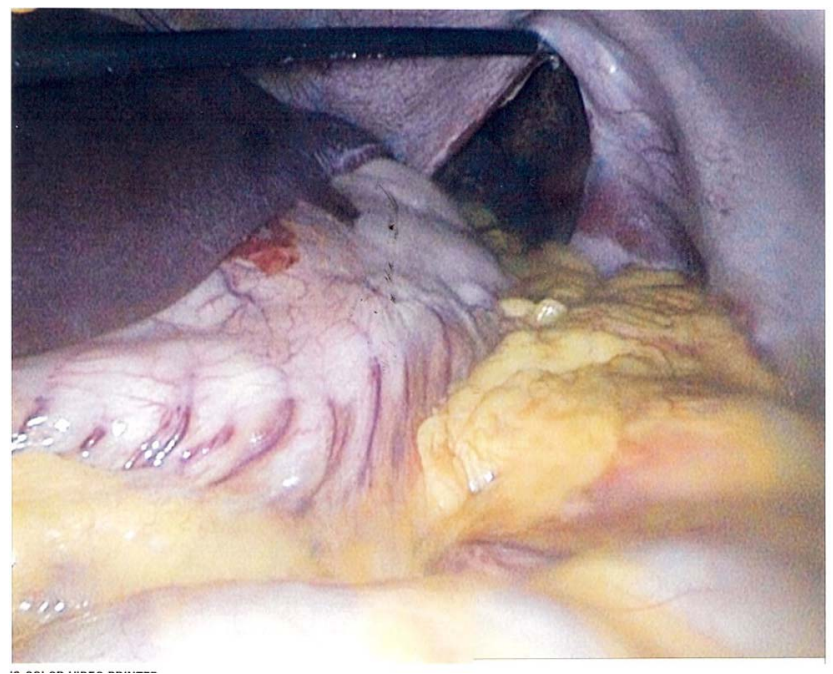

Figure 1. Elevation of the left hemidiaphragm with a 5-mm trocar blunt grasper, allowing complete evaluation of the bluntforce injury to the diaphragm. The lung was without injury, but reduction of the spleen revealed a grade I injury. 


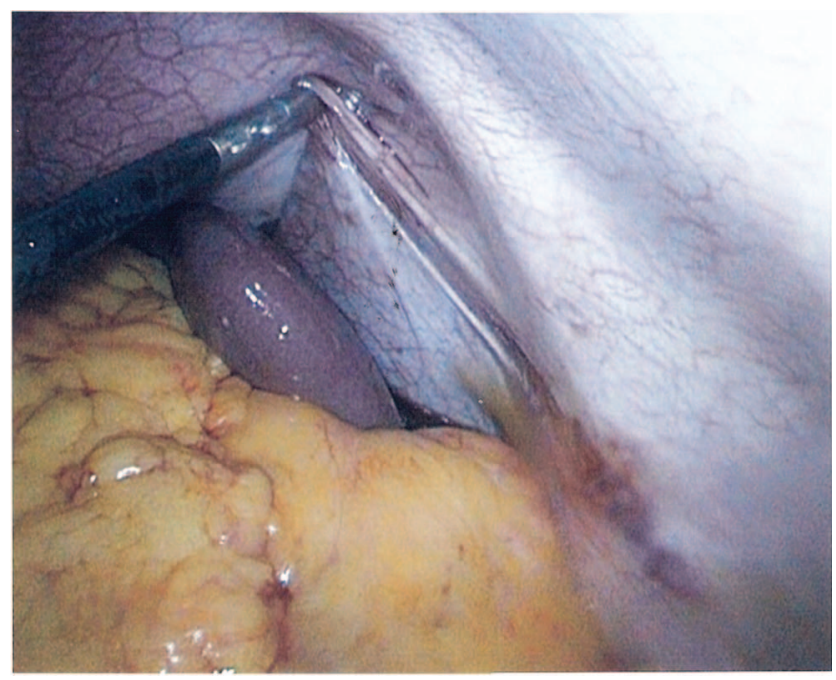

Figure 2. Debrided leaflets of the left diaphragm held in apposition, demonstrating a tension-free repair without mesh placement and intra-abdominal reduction of the spleen.

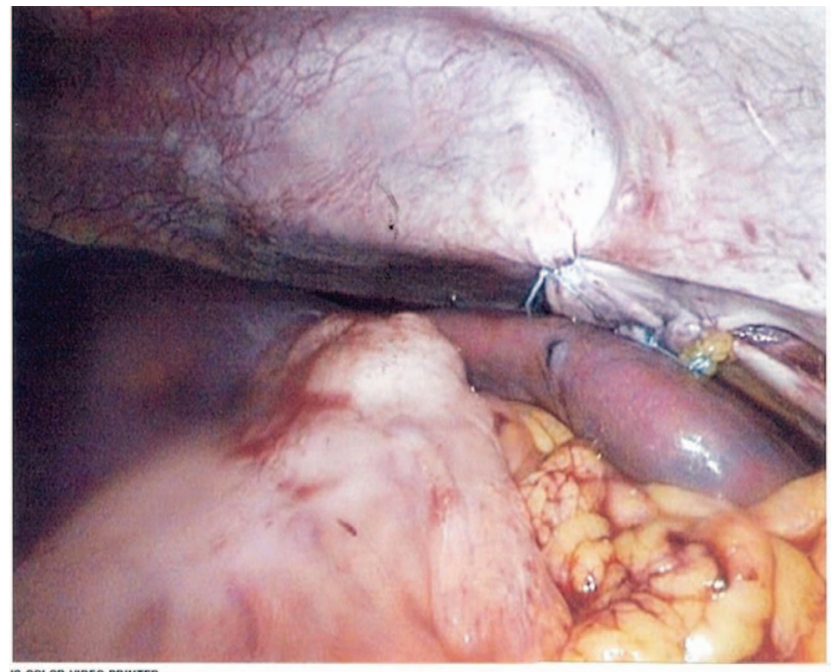

Figure 3. Repair of defect with the abdominal contents returned to the abdominal cavity and no evidence of further splenic injury or bleeding.

sutures. A 10-mm Endostitch device (Covidien, Mansfield, MA, USA) was used in this case, because the teeth located in each jaw allowed both leaflets of the retracted diaphragm to be held in apposition while simultaneously throwing the interrupted sutures. We elected to tie the knots in an extracorporeal fashion. A tension-free repair of the left hemidiaphragm was completed without bleeding or further injury to the spleen (Figure 3). A red rubber catheter can be used to remove air and carbon dioxide from the thoracic cavity, but we opted for the placement of a left chest tube, which we deemed to be the most conservative treatment option.

\section{DISCUSSION}

Blunt-force traumatic rupture of the diaphragm is associated with a high-energy impact to the torso and can lead to herniation of abdominal structures into the thorax. Approximately $75 \%$ of diaphragm injuries occur on the left side, and the most common cause of blunt-force rupture of the diaphragm is a high-speed accident. ${ }^{7-9}$ In the less severely injured patients, possible signs and symptoms include shoulder pain, epigastric pain, vomiting, dyspnea, absent breath sounds, or bowel sounds heard during auscultation of the chest. ${ }^{10}$ Concomitant injuries can also occur as a consequence of blunt-force trauma and may be distracting, leaving the diaphragm injury undiagnosed in the acute phase. The initial diagnostic study is a chest $\mathrm{x}$-ray, with sensitivity reported to be in the range of $27 \%$ to $62 \%$ for left-side injuries. The pathognomonic finding for blunt-force rupture of the diaphragm is visualization of a hollow viscus structure above the level of the diaphragm. ${ }^{11}$ Helical CT for the detection of blunt-force diaphragm disruption has a reported sensitivity of $71 \%$ to $100 \%$ and specificity of $75 \%$ to $100 \%$; sensitivity improves to $78 \%$ to $100 \%$ if only left-side injuries are included. ${ }^{12}$

The treatment of diaphragm disruption is surgical and classically involves a laparotomy in the acute setting and a thoracotomy in the chronic presentation. ${ }^{13}$ Surgical repair of the diaphragm can be challenging, especially in the presence of rib fractures, pulmonary contusions, pneumothorax, or abdominal contents that have herniated into the thoracic cavity compromising respiratory status. Associated trauma injuries with severe hemorrhage or abdominal solid-organ bleeding and contamination from viscus perforation may worsen the morbidity of a ruptured diaphragm. It is for the above-mentioned clinical presentations that open repair of injuries to the diaphragm is the mainstay of treatment in the trauma literature. ${ }^{4}$ Meyer et $\mathrm{al}^{14}$ have reported comparable success in repairing laparoscopically blunt-force diaphragmatic hernias in 3 patients. In their review, they put forth the same caveat of performing laparoscopic procedures in patients who are hemodynamically stable and of thoroughly evaluating the abdomen for the presence of concomitant injuries. Our experience also showed that when the patient is stable and after the appropriate imaging studies, the laparoscopic approach allows for an excellent survey of the polytraumatized abdomen and repair of the injured diaphragm. 


\section{CONCLUSION}

Traumatic rupture of the diaphragm can go unnoticed in the acute phase of trauma and, in the chronic setting, manifests with symptoms of respiratory difficulties, intestinal obstruction, and strangulation of the abdominal viscera. The gold standard, if the diaphragm is traumatically ruptured, is an open survey of the abdomen with open repair of the diaphragm. Our laparoscopic repair of the diaphragm avoided the need for an emergency laparotomy while still allowing surveillance of concomitant injuries (splenic laceration). The avoidance of an open trauma laparotomy aided in the timely recovery of our patient, and at 2-year follow-up, she had no respiratory or abdominal symptoms.

In the correct clinical setting, laparoscopic repair of a blunt diaphragm rupture can be the procedure of choice.

\section{References:}

1. Hsee L, Wigg L, Civil I. Diagnosis of blunt traumatic ruptured diaphragm: is it still a difficult problem? ANZ J Surg 2010;80:166168

2. Matsevych OY. Blunt diaphragmatic rupture: four years' experience. Hernia. 2008;12:73-78.

3. Latic F, Delibegovic S, Latic A, et al. Laparoscopic repair of traumatic diaphragmatic hernia. Med Arh. 2010;64:121-122.

4. Murray JA, Demetriades P, Asensio JA, et al. Occult injuries to the diaphragm: prospective evaluation of laparoscopy in penetrating injuries to the left lower chest. J Am Coll Surg. 1998;187: $626-630$.
5. Powell BS, Magnotti LJ, Finnell CW, et al. Diagnostic laparoscopy for the evaluation of occult diaphragmatic injury following penetrating thoracoabdominal trauma. Injury. 2008;39:530534.

6. Frantzides CT, Carlson MA. Laparoscopic repair of a penetrating injury to the diaphragm: a case report. J Laparoendosc Surg. 1994;4:153-156.

7. Scharff JR, Naunheim KS. Traumatic diaphragmatic injuries. Thorac Surg Clin. 2007;17:81-85.

8. Kearney PA, Rouhana SW, Burney RE. Blunt rupture of the diaphragm: mechanism, diagnosis, and treatment. Ann Emerg Med. 1989;18:1326-1330.

9. Schneider C, Tamme C, Scheidach, et al. Laparoscopic management of traumatic ruptures of the diaphragm. Langenbecks Arch Surg. 2000;385:118-123.

10. Hanna WC, Ferri LE. Acute traumatic diaphragmatic injury. Thorac Surg Clin. 2009;19:485-489.

11. Mirvis SE, Shanmuganagthan K. Imaging hemidiaphragmatic injury. Eur Radiol. 2007;17:1411-1421.

12. Sliker CW. Imaging of diaphragmatic injuries. Radiol Clin North Am. 2005;44:199-211.

13. Kishore GSB, Gupta V, Doley RP, et al. Traumatic diaphragmatic hernia; tertiary centre experience. Hernia. 2010;14:159164.

14. Meyer G, Huittl TP, Hatz RA, et al. Laparoscopic repair of traumatic diaphragmatic hernias. Surg Endosc. 2000;14:10101014 . 